\title{
Present status and issues of control technology for cracking of mass concrete in Japan
}

\author{
Toshiaki Mizobuchi ${ }^{1 *}$, Shingo Asamoto ${ }^{2}$ \\ ${ }^{1}$ Department of Civil and Environmental Engineering, Hosei University, Tokyo, Japan \\ ${ }^{2}$ Department of Civil and Environmental Engineering, Saitama University, Saitama, Japan
}

Received: 27 January 2021 / Accepted: 06 April 2021 / Published online: 03 May 2021

(C) The Author(s) 2021. This article is published with open access and licensed under a Creative Commons Attribution 4.0 International License.

\begin{abstract}
This paper presents the current state of the control technology for cracks caused by heat generation due to the hydration of cement during construction in Japan. For the prediction analysis for thermal cracking of mass concrete, this paper presents the CP method that was developed for the first time in the world, the thermal cracking probability, the simple evaluation method that can predict and estimate the thermal cracking without performing complicated analysis and the evaluation method for expansive materials using the mechanical energy conservation hypothesis. In addition, issues are introduced for the next revision of the guidelines.
\end{abstract}

Keywords: Mass concrete; Thermal crack; Crack control; Volume change; Thermal and mechanical properties

\section{Introduction}

Since 1960, numerous mass concrete structures such as long bridges, nuclear power plants, and underground tanks have been constructed in Japan. In their construction, cracking caused by heat generation due to hydration of cement is one of the major problems [1-4]. Regarding this problem, researchers in Japan carried out academic studies, experimental and analytical, and provided a practical estimation formula for cracking based on measurement results on-site $[5,6]$. The estimation formulas explained the importance of not only the internal restraint of the central part due to heat generation but also the external restraint from the restraint body. In the United States, where the construction of huge structures such as dams was more advanced than in Japan, in 1973, ACl Committee 207 published a report, "Effect of restraint, volume change, and reinforcement on cracking of massive concrete" [7]. In this report, a practical design concept and calculation methods for the control of thermal cracks considering the influence of internal and external restraints are proposed. Until the early 1980s, the formula proposed by ACl 207 Committee has been also widely used in Japan. Considering this background, in the Standard Specifications for Concrete Structures 1974 Fiscal Year Version (Execution), although a chapter on mass concrete has been provided for the first time, measures for thermal cracking have not been described [8]. In this specification, it has only been stated to refer to the measures against thermal cracking in existing concrete dam structures. In the case of other mass concrete structures such as large bridge foundations, as there are many differences from dam concrete, such as mix proportions and construction methods, the countermeasures for thermal cracking in concrete dam structures cannot be transferred directly to those for the target mass concrete structures.

Under such circumstances, JCl Technical Committee on Cracks of Concrete Structures has been established at Japan Concrete Institute in 1981 [9, 10] and 'Guidelines for Control of Cracking of Mass Concrete' has been published in 1986 [11]. This guideline provided a compilation of basic ideas such as control plans of thermal cracking for mass concrete structures, analytical methods for estimation of temperature, thermal stress, and crack width, and management methods. It has been explained that various analytical methods have been proposed through the pioneering research in Japan to predict the temperature increase due to heat of hydration and accompanying thermal stress for the thermal properties, mechanical properties, and internal and external restraint conditions. The analysis method in this guideline is different from that of $\mathrm{ACl}$. In particular, a new concept of the compensation plane (CP) method, developed as a convenient method for estimation of thermal stress, was introduced when a concrete member was directly placed on rock, ground, or existing concrete foundation [12]. The CP method is explained later in detail. In October 1986, the chapter on

\footnotetext{
* Corresponding author: Toshiaki Mizobuchi, E-mail: mizobuch@hosei.ac.jp
} 
mass concrete in the Standard Specification for Concrete Structures (Japan Society of Civil Engineers) has been significantly revised, including the thermal stress analysis method by the CP method, and the relationship between the thermal cracking index (= tensile strength/thermal stress) and thermal cracking probability based on the experimental and existing structure data [13]. The concept of thermal cracking probability is practically useful because cracking can contain many uncertainties such as construction quality, environmental conditions, and material characteristics (Section 2.2 provides detailed information). In addition, an advantage of the assessment for thermal cracking in Japan is the absence of a general temperature limitation; the local cracking possibility can be analysed according to the thermal cracking index in each case individually without the need of the temperature limitation. Other designs generally specify the temperature difference between the inside and surface and maximum temperature $[14,15]$.

The guidelines for the control of thermal cracking have been revised in 2008 for the first time in 22 years because the prediction and countermeasures by the conventional methods became insufficient owing to the remarkable development of design, materials, and construction technology [16]. In the revision, plans, designs, verification, and inspections that have not been always linked together are systematised and the guidelines are based on performance verification. In this guideline, the research results of autogenous shrinkage of concrete by Tazawa et al. $[17,18]$ were incorporated for the first time. It was possible to comprehensively incorporate volume changes due to heat generation with the initial hydration of cement by formulating the strain of autogenous shrinkage considering the type of cement, including blended cement, water-to-cement ratio, and temperature history. Owing to the large improvements in the computing capacities and speeds of personal computers, large-capacity calculations have become relatively simple and thus the control technology of thermal cracking using the finite-element method (FEM) has been widely used in practice in Japan. In addition, the Standard Specification for Concrete Structures 2012 Fiscal Year Version also presented the control method for cracking based on a three-dimensional finite-element method (3D FEM) [19]. Furthermore, in the guidelines for control of cracking of mass concrete revised in 2016, in addition to the update of the control technology, to prevent expansion and cracking by delayed ettringite formation (DEF) that may occur due to the action of hightemperature history, the assessment for DEF cracking risk was incorporated based on the maximum temperature and chemical composition of cement and cement type [20]. Therefore, the addition of verification for the DEF cracking risk has provided more systematic guidelines. The DEF analysis has been recently focused on Asian countries under hot climate [21, 22], while the recommendations for preventing DEF have been proposed in France earlier [23]. Difficulties in identifying DEF cracking are the similarity of the apparent cracking pattern to that of (Alkali Silica Reaction (ASR) and the observed ettringite by microscope in core concrete related to both ASR and DEF [24]. As no cases of DEF cracking have been clearly confirmed in Japan, it would be necessary to further discuss the possible DEF risk in future revisions based on the experience and updating research in Asia and Europe.

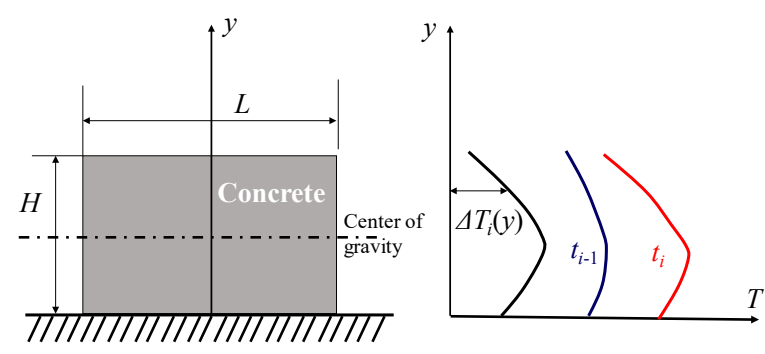

Rock or soil foundation

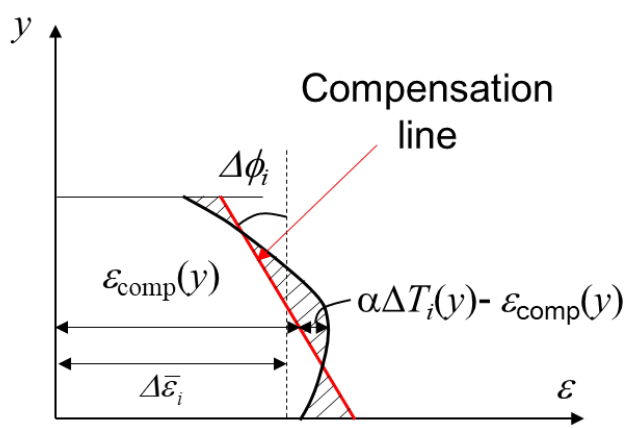

Figure 1. Compensation line.

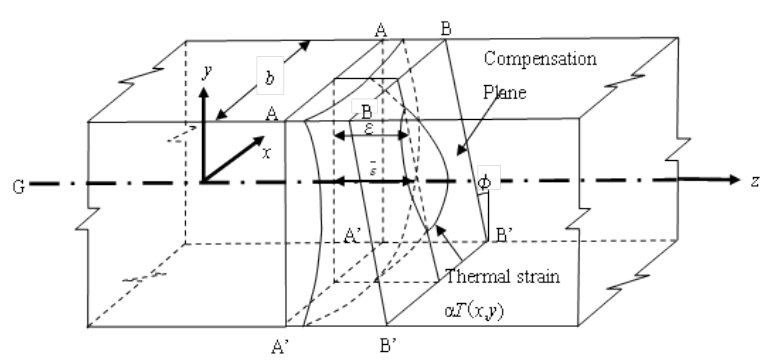

Figure 2. Concept of $\mathrm{CP}$.

Studies are underway on reviewing and clarifying newly added items and contents toward the revision of the guidelines scheduled to be published in 2023. In the next revision, the probability of cracking, design values such as cracking strength, creep at an early age, and concrete temperature at placement will be reviewed. In addition, the application of the crack width analysis method and evaluation method for expansive materials using a type of energy conservation law will also be considered.

This paper presents the control technology for the cracking of mass concrete in Japan and outlines the current issues and future prospects. The detailed information to determine thermal/aging mechanical properties and to involve the effect of shrinkage, creep and others on thermal stress analysis can be referred in the guidelines $[16,20]$. 


\section{Control technology for cracking originated in Japan}

\subsection{CP method}

The CP method developed in Japan can be used to easily predict the thermal stress generated in mass concrete, by assuming that the law of plane retention (linear distribution of strain) is valid within the cross section of a mass concrete structure subject to internal and external restraint in concrete of early age that has not yet sufficiently hardened [12].

\subsubsection{Formulation of the CP method [12]}

As shown in Fig. 1, when the temperature change $T(y)$ is generated inside a concrete member that is not subjected to any external restraint, assuming that the coefficient of thermal expansion of concrete is $\alpha$, the thermal strain $\varepsilon(y)$ is $\alpha T(y)$. However, the deformation of the cross section of the concrete member due to the temperature change does not lead to the strain distribution $\varepsilon(y)$, as the temperature distribution and 'plane section remains plane' are valid except near the end of the cross section of the concrete member. Therefore, the vertical cross section $A A^{\prime}$ before the temperature change becomes $B B^{\prime}$ after the deformation due to the temperature change. $B B^{\prime}$ is the compensation line. In the thermal stress analysis, the compensation line is used in the two-dimensional field and the CP is used in the threedimensional field. Deformations due to the temperature change can be classified into axial and flexural deformations. The mean value $\bar{\varepsilon}$ of the distance between $A A^{\prime}$ and compensation line indicates the axial deformation, while the inclination $\phi$ of the compensation line indicates the flexural deformation. The compensation line determined by $\varepsilon$ and $\phi$ can be obtained from the thermal strain $\alpha T(y)$. After the deformation of the target concrete member, the axial force $N$ and flexural moment $M$ with respect to the centre of gravity $G$ of the residual stress (internal restraint stress) can be expressed as

$$
\begin{aligned}
& N=\int \varepsilon E d y-\bar{\varepsilon} E H=\int \alpha T(y) E d y-\bar{\varepsilon} E H, \\
& M=\int \alpha T(y) E y d y-\int E \phi y^{2} d y,
\end{aligned}
$$

where $y$ is the distance from the centre of gravity and $E$ is the Young's modulus of the concrete.

As shown in Fig. 2, because no external restraint is applied to the member, the following equations can be obtained from the equilibrium between the axial force and flexural moment with respect to the centroid axis of the cross section,

$$
\begin{aligned}
& N=\int_{A} \alpha T(x, y) E s A-\bar{\varepsilon} \cdot E A=0, \\
& M=\int_{A} \alpha T(x, y) E y d A-\int b E \phi y^{2} d y=0, \\
& \bar{\varepsilon}=\frac{\int_{A} \alpha T(x, y) E d A}{E A}, \phi=\frac{\int_{A} \alpha T(x, y) E y d A}{\int b E \varphi y^{2} d y},
\end{aligned}
$$

where the depth of the concrete member is $b$, while the crosssection is $A$.

When the deformation of the concrete member is completely restrained, it is considered that a cross-sectional force of $N=\bar{\varepsilon} E A, M=\int b E \varphi y^{2} d y$ is generated in the member.
When the external restraint does not act, $N=0$ and $M=0$, and the existing structures subjected to the external restraint can be between those states. Therefore, it is possible to obtain the thermal stress by appropriately evaluating the effect of the external restraint.

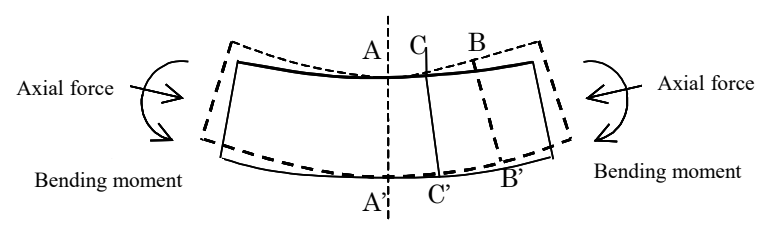

Figure 3. State of the external restraint.

\subsubsection{Concept of coefficient of external restraint in the CP method [25]}

In most cases, existing concrete structures are constrained by restraint bodies such as bedrock at the construction site. The dotted line in Fig. 3 shows the deformation of the concrete member when only the internal restraint is applied. However, when the targeted member is subjected to the action of external restraint, it does not exhibit as large deformation as that of the dotted line, but only the deformation shown by the solid line. In Fig. 3, the axial force and flexural moment required to return the compensation line $B B^{\prime}$ to the fully restrained state $A A^{\prime}$ are defined as the fully restrained axial force and fully restrained flexural moment, respectively. When the concrete member is constrained in a state where it is not completely restrained, because the deformed state is an intermediate state, as shown by the solid line in Fig. 3, the position of the compensation line moves from $B B^{\prime}$ to $C C^{\prime}$. It can be considered that the compensation line $B B^{\prime}$ moved horizontally by the axial force $N$, and then moved to $C C^{\prime}$ by rotating because of the flexural moment $M$.

This amount of movement depends on the difference in stiffness between the restraining body and target concrete member. The coefficients of external restraint $R_{N}$ and $R_{M}$ are introduced to express the degree of restraint. The ratios of the actual acting axial restraint force and restraint flexural moment to the perfect restraint axial force and perfect restraint flexural moment are defined as the coefficient of axial restraint and coefficient of flexural restraint, respectively, expressed as

$R_{N}=\frac{N}{N_{0}}, \quad R_{M}=\frac{M}{M_{0}}$,

where $R_{N}=0$ and $R_{M}=0$ indicate the state in which only the internal restraint is applied, while $R_{N}=1$ and $R_{M}=1$ indicate full restraint. In general, $R_{N}$ and $R_{M}$ are values between 0 and 1 when the target concrete member is restrained. However, as the axial force and flexural moment are also generated in the restraint, such as rock foundations, by a temperature change, the axial force and flexural moment in the concrete member may be larger than those of the full restraint. In this case, each restraint is $R_{N}>1$ and $R_{M}>1$.

The restraint coefficients can be determined depending on the nomograms of $L / H$ (Ratio of the bottom length to the height of the structure) and $E_{c} / E_{r}$ (Ratio of the concrete stiffness to the restraining body stiffness). The detailed 
explanation in English to determine the coefficients can be referred in the English version of Standard Specification for Concrete Structures 2007 (Japan Society of Civil Engineers) [26].

By introducing the external restraint coefficient, the external restraint axial force and external restraint flexural moment can be defined. Therefore, the external restraint stress $\sigma_{R}$ can be calculated using the external restraint axial force, external restraint flexural moment, and external restraint coefficient,

$\sigma_{R}=\frac{R_{N} N_{0}}{A}+\frac{R_{M} M_{0}}{I} y$,

where $\sigma_{R}$ is positive on the compression side and $l$ is the moment of inertia of the area.

\subsubsection{Crack width analysis using the CP method}

As the CP method is used to verify whether initial cracks occur, it is not used to determine the crack width progression after cracking. Hence, the CP crack width method, which is one of the methods for estimating the crack width upon cracking, is described $[27,28]$. The CP crack width method extends the concept of the CP method after cracking and applies the assumption of plane section hypothesis to the strain of the reinforcing bar in the cross section of cracking and concrete strain around the crack. It is an analysis method for calculating the thermal crack width and concrete stress after cracking using the force balance and strain of conformity conditions. In the CP crack width method, assuming that the concrete stress is released with the occurrence of cracks and a part of it is taken over by the reinforcing bar, it is determined by the area where the concrete stress is released, length representing the bond characteristics of the reinforcing bar, and assumption of plane section hypothesis. It is assumed that the cracks reach from the bottom surface to the top surface of the target member because the CP crack width method is applied to the structure whose wall length is longer than the height of wall and combines beam bending and axial force. Therefore, it is not suitable for applications with small $L / H$. As shown in Fig. 4, as the release area of concrete stress due to cracks, two regions, the area where there is no adhesion between the reinforcing bar and concrete (bond loss equivalent area; $I_{s}$ ) and area where the boundary surface between the reinforcing bar and concrete is peeled off (stress release area; $I_{c}$ ), are assumed in the CP crack width method. The crack width can be calculated by the following steps.

Step 1: Immediately after the cracking, the balance of force between the reinforcing bar inside the concrete member and cracked concrete is obtained without deforming the restraint. The stress release region boundary in Fig. 4 is fixed by carrying out step 1.

Step 2: The fixed boundary of the energy release region shown in Fig. 4 is loosened and the CP method is applied to the cracked cross section and cross section including the restraint.

Step 3: When a temperature change occurs after a certain period of time has passed since the crack occurred, as in step 1 , the CP method is applied to the balance of the force between the reinforcing bar inside the member and cracked concrete without deforming the restraint and the stress redistribution within the stress release region is obtained.

Step 4: When a temperature change occurs after a certain period of time has passed since the crack occurred, as in step 2 , the CP method is applied to the cracked cross section and cross section including the restraint to release the fixation of the stress release region.

In steps 1 and 2, the CP method is applied twice to each cross section of the cracked member and cross section including the target member and restraint. In the cracked cross section, it is a prerequisite that the boundary surface between the restraint body and target member is peeled off and that the assumption of plane section hypothesis is established in each cross section. Therefore, the strain distribution over the entire cross section is discontinuous on the upper surface of the restraint. In addition, as steps 3 and 4 are used to obtain changes in the crack width due to temperature changes after cracking, the calculation method is the same as in steps 1 and 2.

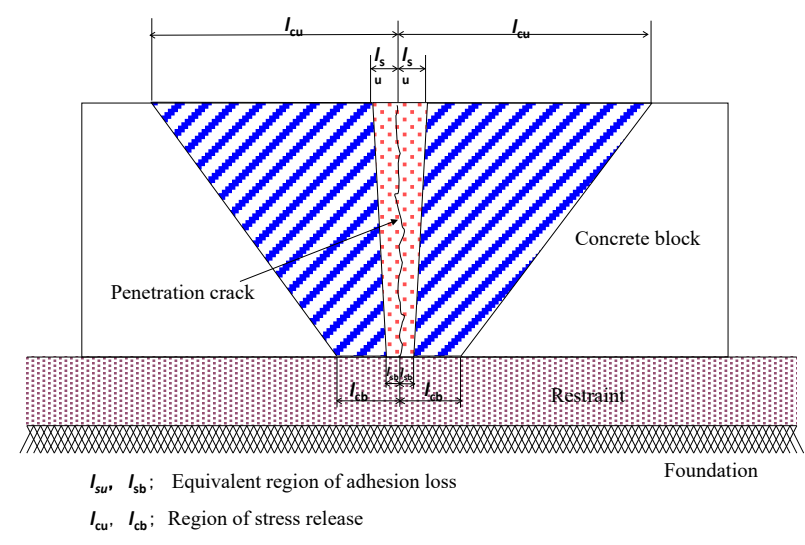

Figure 4. Outline of the analysis model in the CP crack width method.

\subsection{Relationship between the thermal cracking index and thermal cracking probability}

The thermal cracking probability is a judgement index that indicates the cracking tendency stochastically when the tensile stress in concrete is induced by both autogenous shrinkage and volumetric change of concrete due to heat of hydration and is generally expressed in relation to the thermal cracking index. The thermal cracking index is defined as the ratio of the tensile strength to the stress in some elements in the concrete member. The stresses in concrete are calculated by the numerical analysis method and the tensile strength of concrete is obtained by the applied testing method. Therefore, the thermal cracking probability depends on the analysis method and strength test method.

In Guidelines for Controlling the Cracking of Mass Concrete 2008, the relationship between the thermal cracking index and thermal cracking probability is recommended to be based on the most popular numerical method considering the numerical conditions that meet the current technical needs $[14,20]$. A 3D FEM is used for temperature and stress analyses, in which input models are applied for the physical 
properties of early-age concrete and foundations that can simulate the thermal and mechanical behaviours and in which the element and boundary conditions of the numerical model that engineers can usually use are shown. These models are specified in the guidelines. In the analysis for estimation of temperature and thermal stress, the design values of the material properties of concrete, steel, ground/bedrock and other materials, such as compressive and tensile strengths, Young's modulus, thermal properties and others are used.

In the revisions of Guidelines for Control of Cracking of Mass Concrete 2008, a review of the relationship between thermal cracking probability and thermal crack index has been carried out, where 65 structures have been chosen. They have an adequate information on the shape and size of the structure, construction conditions such as the date of concrete casting, materials used for concrete, and mix proportions. The obviously inadequate data have been excluded and 728 data points were available for the analysis. The number of data points on the structures with cracking was 204. The number of data points on the structures without cracking was 524. The detailed data classification according to the type of member is as follows: the amounts of data on wall, slab, multi-layered members, and column structures are $282,63,349$, and 34 , respectively.

The suitable probability distribution of thermal cracking was examined according to the results of the questionnaire and 3D thermal stress analysis. In Guidelines 2008, the adaptability of the Weibull distribution function as a regression curve of the thermal cracking probability has been analysed. The Weibull distribution function suggested in 1939 by the Swedish physicist Weibull is the probability density distribution function, derived based on the idea that the strength of the material depends on that at the weakest part and used to express the failure rate of the products.

The Weibull distribution function is an exponential function. The probability density function and cumulative distribution function are

$$
\begin{aligned}
& f(x)=\lambda \exp (-\lambda x), \\
& F(x)=\int_{0}^{x} f(x) d x=1-\exp (-\lambda x),
\end{aligned}
$$

When the time dependency of Eq. (8) is considered, the probability density function and cumulative distribution function are expressed as

$$
\begin{aligned}
& f(x)=\frac{m}{\alpha} x^{m-1} \exp \left(-\frac{x^{m}}{\alpha}\right), \\
& F(x)=1-\exp \left(-\frac{x^{m}}{\alpha}\right)
\end{aligned}
$$

Application of natural logarithm to both sides of Eq. (9) twice yields

$\ln \left[\ln \left(\frac{1}{1-F(x)}\right)\right]=m \ln x-\ln \alpha$,

The correlation between the thermal cracking probability and thermal cracking index, both expressed by the natural logarithm in Eq. (10), is very high. Thus, the regression analysis using the Weibull distribution function is applied to the data based on the frequency of cracking or no cracking in each field structure and then the constants, that is $m$ and $\alpha$, are decided to be

-4.29 and 0.92 , respectively. The analysed results are shown in Fig. 5.

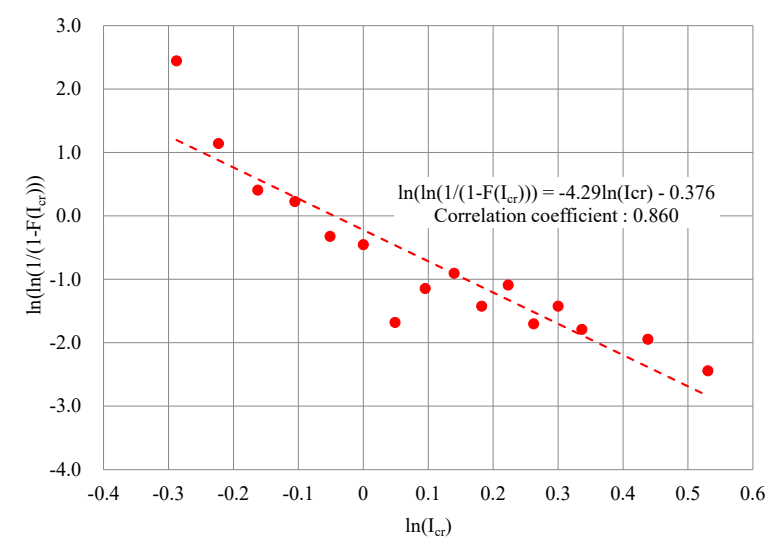

Figure 5. Relationship between thermal cracking index and thermal cracking probability.

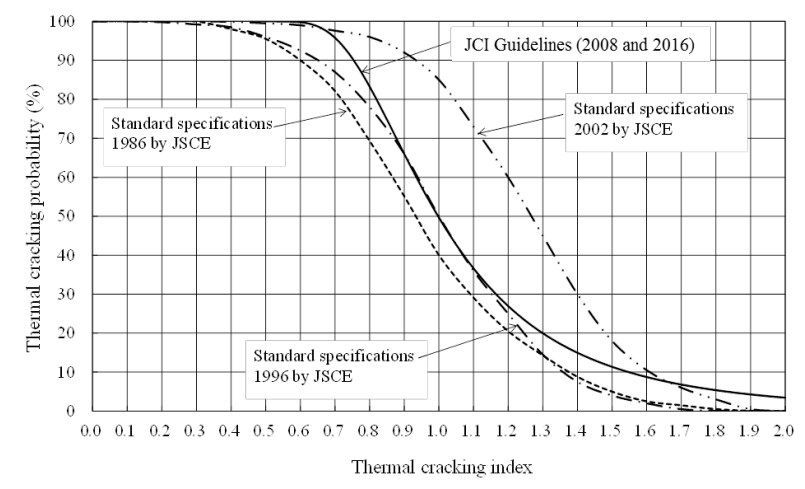

Figure 6. Thermal cracking probability.

The equation for the relationship between the thermal cracking probability and thermal crack index is obtained below in this guidelines applying $m=-4.29$ and $\alpha=0.92$ to equation (9).

$F\left(I_{c r}\right)=1-\exp \left(-\frac{I_{c r}{ }^{-4.29}}{0.92}\right)$

where $I_{c r}$ is the thermal cracking index and $F\left(I_{c r}\right)$ is the thermal cracking probability.

Figure 6 compares the probability curve based on the Weibull distribution function to the probability curves of normal distributions based on past standard specifications for concrete structures by Japan Society of Civil Engineers (JSCE). As shown in Fig. 6, the inclination of the probability curve based on the Weibull distribution function of the thermal cracking probability in the $\mathrm{JCl}$ guidelines (2008 and 2016) is sharpest.

The probability curve based on the Weibull distribution function, suggested in the JCl Guidelines 2008 and 2016, shifts from the probability curve based on the standard specifications for concrete structures (2002) to the left. When the thermal cracking probability is $50 \%$, the thermal cracking index in the guidelines is smaller than that based on the 
standard specifications for concrete structures (2002) by approximately 0.3 . In the guidelines, the tensile strength should be obtained by the splitting test. The tensile strength in the structure is smaller than that in the splitting test by $20 \%$ to $30 \%$. The thermal cracking index in the guidelines is 1.0 when the thermal cracking probability is $50 \%$. The thermal cracking index in the guidelines can express the actual phenomena considerably better than that based on the standard specifications for concrete structures in 2002. As the temperature in the structures is higher than that in standard water curing, the tensile strength in structures may be the same as that obtained by the splitting test. With these considerations, the thermal cracking index should be overestimated by $10 \%$ to $20 \%$. It is natural that the thermal cracking index should be 1.0 when the thermal cracking probability is $50 \%$.

\subsection{Simple evaluation formula for the thermal cracking index $[14,20]$}

The thermal stress analysis is often carried out in the design and stage before construction. In the analysis, the risk of occurrence of cracks due to thermal stress was evaluated by modelling the target structure. Currently, when the risk of cracking is high, it is necessary to employ measures for the control of thermal cracking. When countermeasures are employed for the control of thermal cracking, changes in used materials, mix proportions, etc., are only changes in design values and can be facilitated. When the number of elements of the analysis model is large or when changing the thickness of the member or lift thickness, considerably more time is needed for the analysis. Therefore, a simplified evaluation formula has been proposed in which approximate values are conventionally calculated using analysis conditions without carrying out a thermal stress analysis. In addition, in the Guidelines for Control of Cracking of Mass Concrete 2008, simplified evaluation formulas have been proposed, classifying into three types of structural forms: wall-like structures, layered structures, and columnar structures. However, the simplified evaluation formula had some deviations in the data such as the type of structure, structure type, used materials, mix proportions, and concrete temperature of placement, and had some restrictions on the scope of application.

In the Guidelines for Controlling the Cracking of Mass Concrete 2016, to expand the applicable range of the simplified evaluation formula for estimating the thermal cracking index, the structure shape, used material, mix proportion, construction method, environmental condition, and curing condition are set as parameters so that there is no deviation of data. A simple evaluation formula was obtained from the results of the thermal stress analysis by the 3D FEM. In the Guidelines for Control of Cracking of Mass Concrete 2016, a simple evaluation formula is applied to two types of structural forms of wall-type structures and structures other than wall-type structures. As a procedure for derivation, a simplified evaluation formula of the thermal cracking index is obtained using the 3D FEM for the analytical models of approximately 4900 structures (wall-type structures: 600 cases, other structures: 4300 cases) with the structure shape, materials, mix proportions, construction method, environmental conditions, and curing conditions as variables.

The factors that are assumed to be strongly related to the causes of thermal cracking are classified into several categories and the coefficient of influence of each category is calculated. The influence factors are classified into three categories: shape and stiffness, used material and mix proportions, and curing method. Each influence coefficient was calculated using a multiple regression analysis by setting the ratio of the objective function and explanatory variable to the case as the basis (referred to as basic case) in each structural form. The estimated value of the thermal cracking index was obtained by multiplying the thermal cracking index of the basic case by the above influence coefficient. A simple evaluation formula for the thermal cracking index was obtained by correcting such that the estimated value of the thermal cracking index matched with the lower limit of the thermal cracking index calculated by the 3D FEM. In this paper, a simple evaluation formula for wall-type structures is given as an example.

For the minimum thermal cracking index of wall-type structures, explanatory variables were set with reference to the results obtained at the time of deriving the simplified evaluation formula of the 2008 guidelines and a simplified evaluation formula was obtained.

Figure 6 shows the relationship between the thermal cracking index obtained by the 3D FEM and thermal cracking index obtained by the estimation formula of Eq. 12 for wall-type structures. The multiple correlation coefficient of each cement type was in the range of 0.812 to 0.986 , which showed a relatively high correlation. In the Guidelines for Control of cracking 2008, a simple evaluation formula was proposed for wall-type structures with wall thicknesses larger than $1 \mathrm{~m}$. However, according to the Guidelines for Control of cracking 2016, even if the application range is expanded to the range of 0.5 to $2.0 \mathrm{~m}$ (wall thickness), a relatively high correlation is obtained and it is possible to estimate the minimum thermal cracking index using a simple evaluation formula,

$I_{m c r s-W T}=\left(\alpha_{1}^{\eta} \cdot \alpha_{2}^{\zeta} \cdot \alpha_{3}^{\xi}\right)^{\beta} \cdot I_{c r c 0}$

$I_{\text {crs }-W T}=I_{m c r s-W T}-I_{b}$,

$\alpha_{1}=a_{0}+a_{1}\left(\frac{1}{D / D_{0}}\right)+a_{2}\left(\frac{1}{L / L_{0}}\right)+a_{3} \ln \left(\frac{H}{H_{0}}\right)+$

$a_{4}\left(\frac{1}{\frac{E_{C} / E_{r}}{E_{C 0} / E_{r 0}}}\right)$,

$\alpha_{2}=b_{0}+b_{1} e^{\left(-\frac{T a}{T_{a 0}}\right)}+b_{2}\left(\frac{Q_{\infty}}{Q_{\infty 0}}\right)+b_{3}\left(\frac{\gamma_{A T}}{\gamma_{A T 0}}\right)+$

$b_{4}\left(\frac{f_{c}{ }^{\prime}}{f_{c 0}{ }^{\prime}}\right)^{0.45}+b_{5}\left(\frac{s_{A T}}{s_{A T 0}}\right)$

$\alpha_{3}=c_{0}+c_{1} \ln \left(\frac{T_{a}}{T_{a 0}}\right)+c_{2}\left(\frac{h}{h_{0}}\right)+c_{3}\left(\frac{t}{t_{0}}\right)+c_{4} e^{\left(\frac{T_{a t}+\Delta T}{T_{a t}}\right)}$,

where $I_{\text {mcrs-WT }}$ is the thermal cracking index of wall-type structures calculated by the multi-regression equation, $I_{b}$ is reduction constant introduced to keep the thermal cracking 
indexes estimated by the simple equation on the safe side as compared with those computed by the 3D-FEM. $l_{b}$ is 0.2 , in principle, $\alpha_{1}^{\eta}$ is the influence coefficient on the shape and stiffness, $\alpha_{2}^{\zeta}$ is the influence coefficient on the used materials and mix proportions, $\alpha_{3}^{\xi}$ is the influence coefficient on curing methods, $\eta, \zeta, \xi$, and $b$ are constants representing the influence of the 'influence coefficient' of each cement on the thermal cracking index, $I_{\text {croo }}$ is the basic thermal cracking index for each type of cement (the basic value is 1.00), $I_{\text {crs- } w \text { T }}$ is the thermal cracking index of wall-type structures obtained by a simple estimation formula, and $a_{0}$ to $a_{4}, b_{0}$ to $b_{5}$, and $c_{0}$ to $c_{4}$ are constants of the regression equation for each type of cement.

Eq. 12 and Eq. 13 are the multi-regression equation and recommended simple estimation equation, respectively. As Eq. 13 is obtained under certain conditions, it should not be applied if the investigated conditions are beyond the applicable limits.

Figure 7 shows that the thermal cracking indexes calculated by the multi-regression equation agree well with the minimum thermal cracking indexes calculated by the 3D FEM. However, the predicted thermal cracking indexes larger than those calculated by the 3D FEM would not provide verification results on the safe side. Therefore, Eq. 13 derived by subtracting the value $I_{b}$ from the multi-regression equation is recommended as the simple estimation equation. The value of $I_{b}$ should be determined on the safer side so that the thermal cracking index estimated by the multi-regression equation may not exceed that calculated by the 3D FEM.

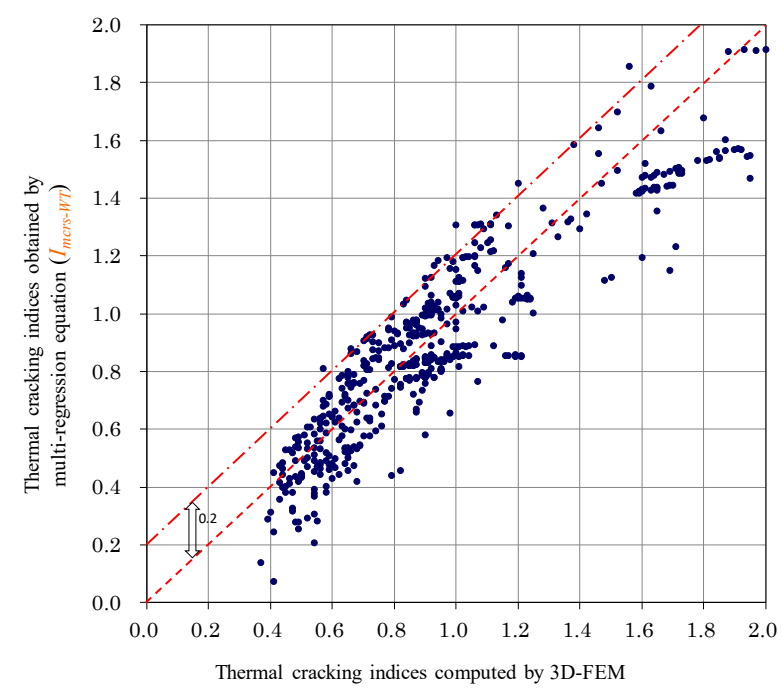

Figure 7. Relationship between the thermal cracking indexes of wall structures obtained by the multi-regression equation and those obtained by the 3D FEM

When $I_{b}$ is 0.2 , as the majority of the thermal cracking indexes estimated by the simple estimation equation do not exceed the minimum thermal cracking indexes calculated by the 3D FEM (Fig. 8), a level of safety equal to or higher than that of the 3D FEM can be ensured. Thus, the value of 0.2 is used for $I_{b}$ in principle. The thermal cracking index calculated by the simple estimation equation may be unnecessarily large under some structural conditions. In such cases, if the results of the application of the simple estimation equation to similar structures have been accumulated and the specific characteristics (evaluation accuracy of the thermal cracking index in relation to the 3D FEM) can be confirmed, $l_{b}$ can be set to a value smaller than 0.2 .

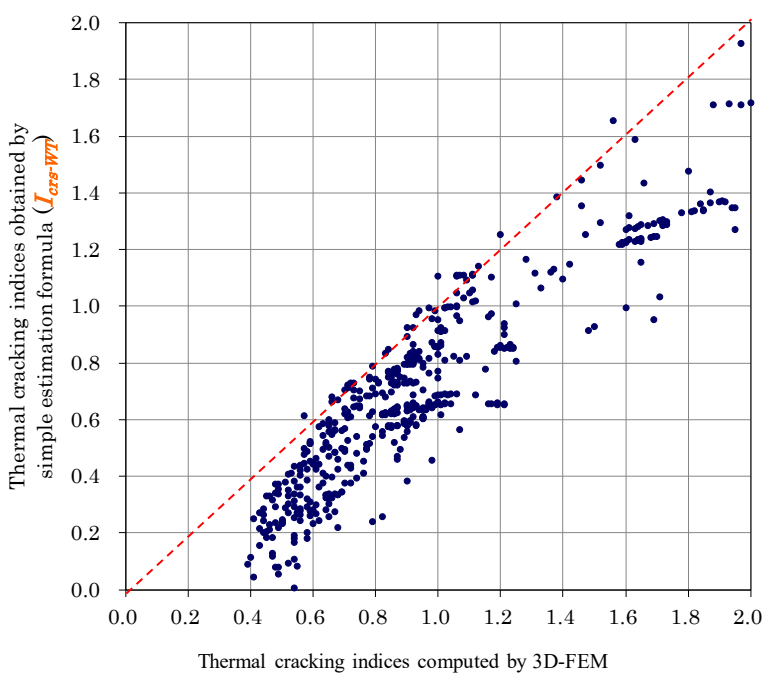

Figure 8. Thermal cracking indexes obtained by the simple estimation formula plotted against results of 3-D-FEM.

\subsection{Formulation of chemical expansion mechanics based on the mechanical energy conservation hypothesis}

In Japan, the initial strain method has been generally used for the problem of cracks caused by the chemical expansion of concrete. The initial strain method was also used in the Guidelines for Control of Cracking of Mass Concrete 2016, as, despite the problems with the application of this method, no alternative method has been proposed. As the chemicalreaction-induced expansion of cement concrete originates from the volume change of the substance formed by the reaction, a method of calculating this expansion mechanism based on energetics has been proposed. To clarify this subject, Prof. Tsuji proposed the concept of work constant theory for expansive cement concrete in 1975 [29]. According to his theory, although, when the reinforcing ratio changes, the initial stress varies depending on the difference in restraint due to the reinforcement ratio, the steel strain energy is almost the same regardless of the steel amount, as shown in Fig. 9.

However, the work constant theory of Y. Tsuji has a deficiency that neglects the work carried out within itself. Prof. Tanabe expanded the theory for general cases and proposed a mechanical energy conservation hypothesis [30-32]. Based on the results of the restraint experiment described above, the mechanical energy conservation hypothesis is explained.

The formulation for the introduction of the concept of the mechanical energy conservation hypothesis into the 3D FEM stress analysis is shown in Fig. 10. 
Re-bar ratio varied from $0.667 \%$ to $4.22 \%$

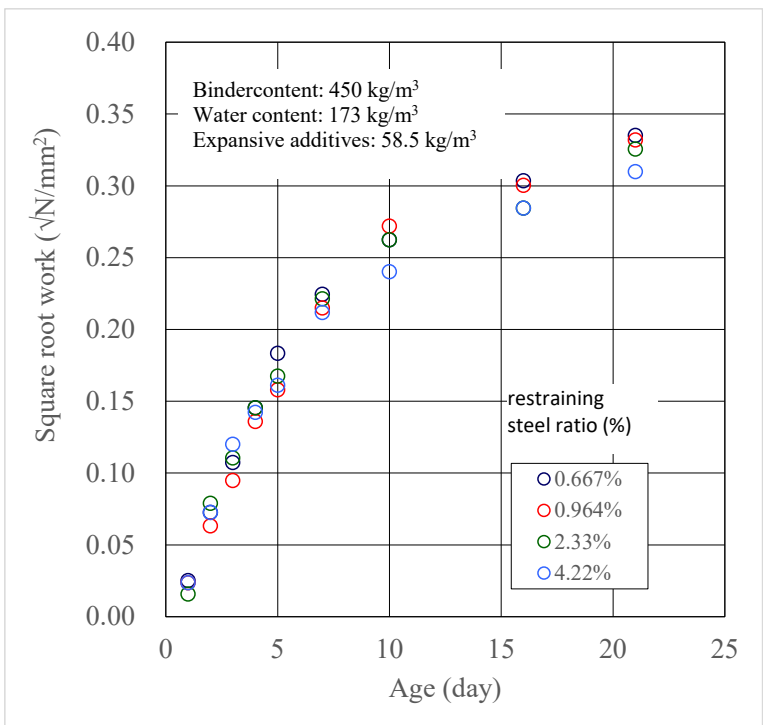

Strain energy in steel
Re-bar ratio varied from $0.667 \%$ to $4.22 \%$

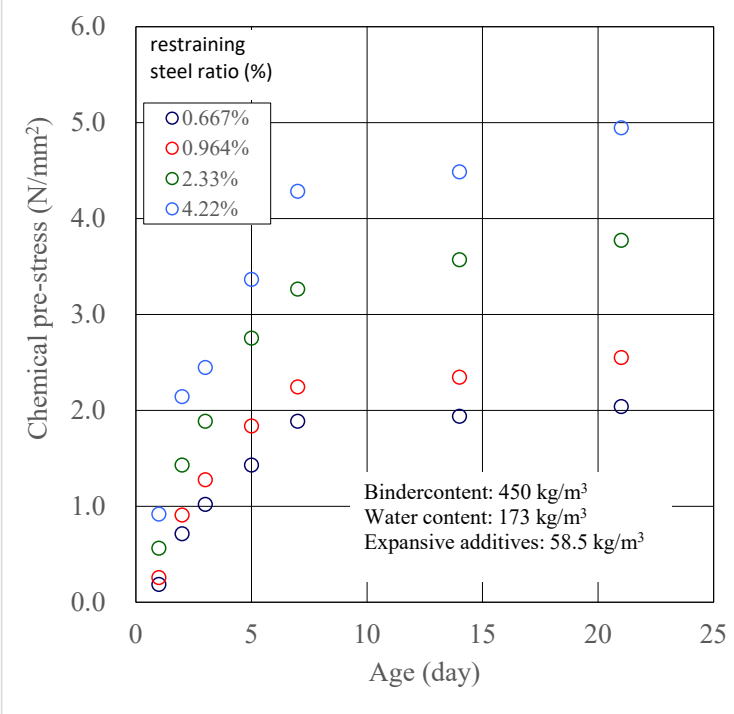

Chemical pre-stress

Figure 9. Concept of work constant theory.

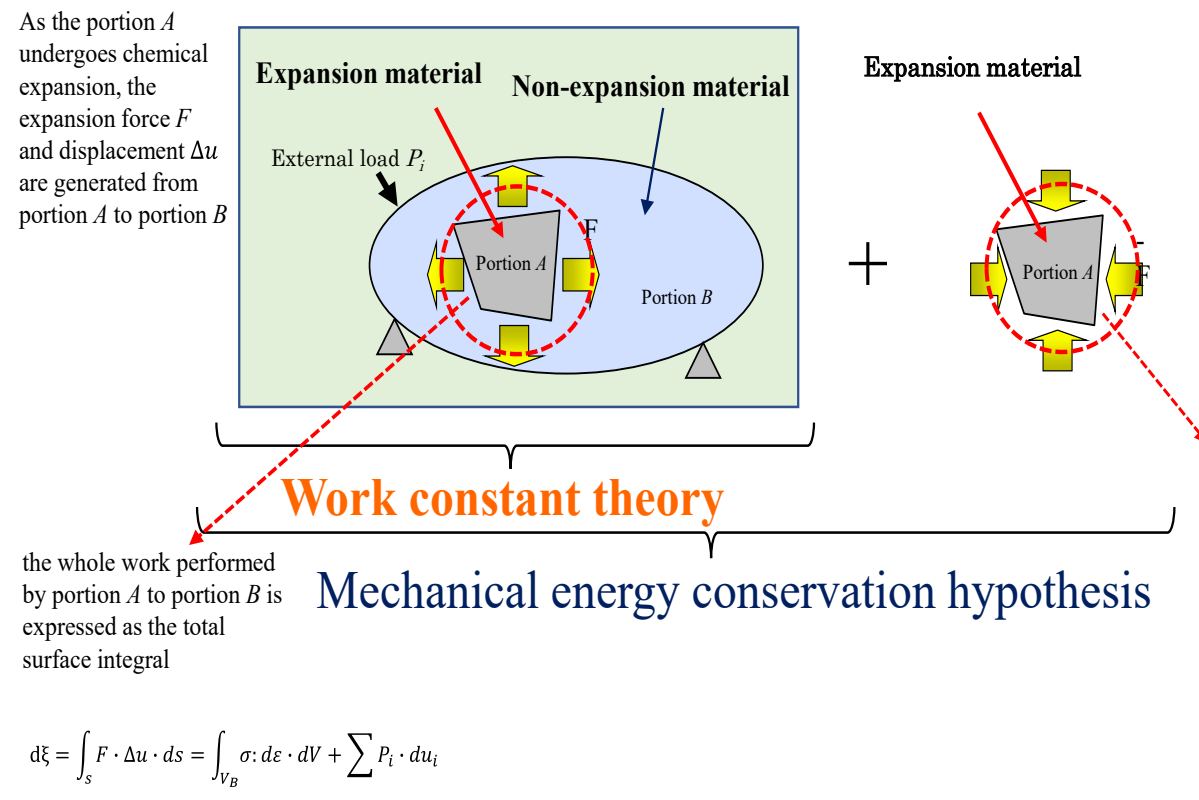

$F$ : Expansion force working at the periphery for portion $B$ (Non-expansion materials) from portion $A$ (Expansion material)

$-F$ : Reaction force working for portion $A$ from portion $B$

Work performed by portion $B$ to portion $A$

$$
-\mathrm{d} \xi=\int_{s}-F \cdot \Delta u \cdot d s=\int_{V_{A}} \sigma: d \varepsilon \cdot d V
$$

Figure 10. Concept of the mechanical energy conservation hypothesis.

As portion $A$ undergoes chemical expansion, the expansion force $F$ and displacement $\Delta u$ are generated from portion $A$ to portion $B$. Therefore, the entire work performed by portions $A$ to $B$ is obtained as the total surface integral. $F$ working at the periphery of portion $B$ from portion $A$ leads to the reaction force $(-F)$, which works for $A$ from $B$ portion. Therefore, the work carried out by $B$ to $A$ portion is $d \xi$, which is equal to the strain energy increment within portion $A$, which is the expanding portion.

$$
\begin{aligned}
& \mathrm{d} \xi=\int_{S} F \cdot \Delta u \cdot d s \\
& \quad=\int_{V_{B}} \sigma: d \varepsilon \cdot d V+\sum P_{i} \cdot d u_{i}
\end{aligned}
$$

$\int_{V_{A}} \sigma: d \varepsilon \cdot d V$

$=\int_{V_{A}} \sigma:\left(d \varepsilon_{e}+d \varepsilon_{c r}+d \varepsilon_{s h r}+d \varepsilon_{c h e}\right) d V$

$=\int_{V_{A}} \sigma: d \varepsilon_{e} d V+\int_{V_{A}} \sigma: d \varepsilon_{c r} d V$

$+\int_{V_{A}} \sigma: d \varepsilon_{s h r} d V+\int_{V_{A}} \sigma: d \varepsilon_{c h e} d V$ 
where portion $A$ is the expansion material, portion $B$ is the nonexpansion material, $d \xi$ is the work carried out by $B$ to $A$ portion, $\Delta \varepsilon$ is the total strain, $\Delta \varepsilon_{\mathrm{e}}$ is the elastic strain, $\Delta \varepsilon_{\mathrm{cr}}$ is the creep strain, $\Delta \varepsilon_{\mathrm{shr}}$ is the drying shrinkage, autogenous shrinkage, and thermal strain, and $\Delta \varepsilon_{\text {che }}$ is the chemical expansion strain.

$$
\begin{aligned}
& -d \xi=\int_{V_{A}} \sigma: d \varepsilon \cdot d V=\int_{V_{A}} \sigma: d \varepsilon_{e} d V \\
& +\int_{V_{A}} \sigma: d \varepsilon_{c r} d V+\int_{V_{A}} \sigma: d \varepsilon_{s h r} d V+\int_{V_{A}} \sigma: d \varepsilon_{c h e} d V \\
& =d \xi_{e}+d \xi_{c r}+d \xi_{s h r}+d M_{c h e} \\
& d M_{c h e}=d \xi_{e}+d \xi_{c r}+d \xi_{s h r}+d \xi
\end{aligned}
$$

Therefore, the chemical expansion energy is the work carried out to the outside system when a chemical reaction occurred. The mechanical energy conservation hypothesis is a theory that extends the constant work theory to concrete other than expanded concrete. In other words, $\mathrm{d} M_{\text {che }}$ is equivalent to $M_{1}$ and $M_{2}$, as mentioned above.

As the easiest approach to calculate the chemical expansion energy, for example, as shown in Fig. 11, if the strain history of steel in uniaxial expansion tests under conditions constrained by steel is obtained, it may be possible to analyse the crack control effect of expansion materials using the mechanical energy conservation hypothesis. The mechanical energy $(\mathrm{Me}$ ) can be used to obtain this equation,

$M e=\frac{1}{2} p \cdot E_{s} \cdot \varepsilon_{s}^{2}+\sum p^{2} \cdot \frac{E_{s}{ }^{2}}{E_{c}} \cdot \varepsilon_{s} \cdot \Delta \varepsilon_{s}$,

The first term on the right side is the work carried out by the expansion concrete to the constrained steel, while the second term on the right side is the work carried out to expand the concrete. This equation assumes that the concrete and steel are elastic bodies; the effect of creep is included in this equation. $p$ is the restraining steel ratio (\%), $E_{s}$ is the Young's modulus of steel $\left(\mathrm{N} / \mathrm{mm}^{2}\right), \varepsilon_{s}$ is the strain of steel (strain measured during the restraint expansion test), and $E_{c}$ is the Young's modulus of concrete.

For example, the chemical expansion energy is calculated using the results of the restraint expansion test with expansive cement concrete, as shown in Fig. 11. As test parameters, the restraining steel ratio is approximately $1 \%$, underwater curing is carried out at $20^{\circ} \mathrm{C}$ for 7 days, the type of cement is ordinary Portland cement, the amount of expansive additive is $20 \mathrm{~kg} / \mathrm{m}^{3}$, and the water-binder ratio is $50 \%$. Figure 11 shows the results for the regression equation based on the measurement results of the restraint expansion strain, while Figure 12 shows the regression equation obtained from the relationship between the Young's modulus of concrete and age. The chemical expansion energy calculated based on the above conditions is shown in Fig. 13.

Using this method, it is possible to simulate the behaviour of concrete using an expansive material. It is considered to introduce this method in the next revision of Guidelines for Control of Cracking of Mass Concrete.

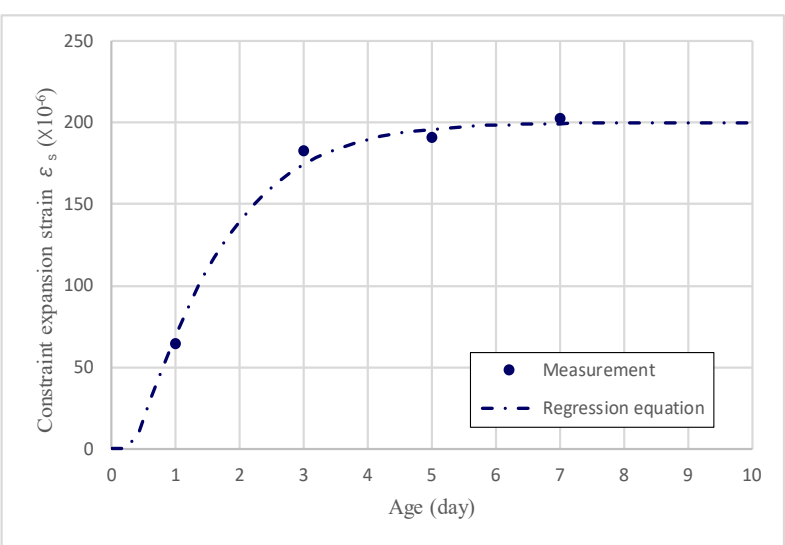

Figure 11. Restraint expansion test.

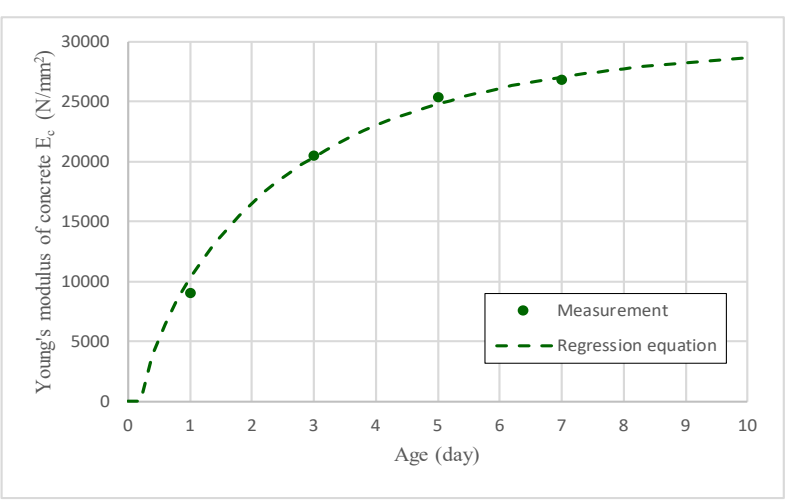

Figure 12. Relationship between the Young's modulus of concrete and age.

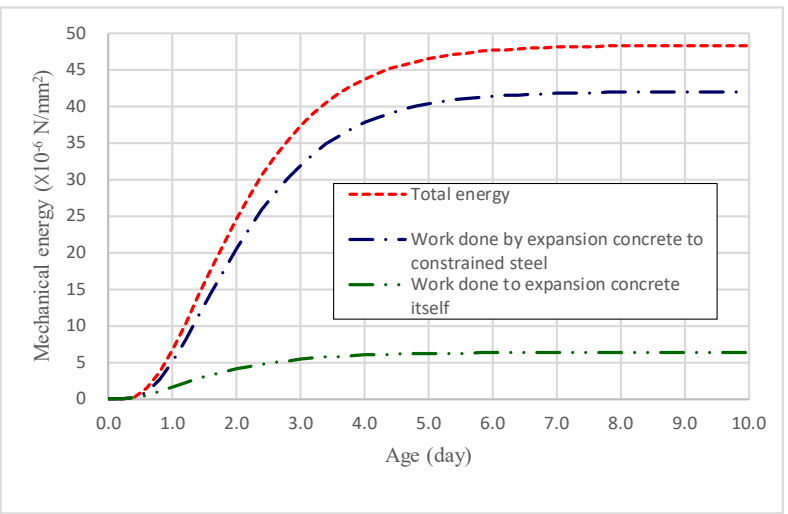

Figure 13. Relationship between the mechanical energy and age.

\section{Introduction of a method that combines the initial stress analysis and load-carrying capacity analysis}

In Japan, the control technology of cracking has been developed for initial stress problems including the thermal stress caused by heat generation with hydration of cement, crack problems caused by autogenous shrinkage, and crack problems caused by drying shrinkage. These problems are time-dependent. To estimate them, it is necessary to solve the differential equations related to time, calculate the initial strain, and solve the initial stress based on the material 
properties at that time. The analysis of load-carrying capacity is a problem in which the material properties do not change with time. If the initial stress problem is an analysis that calculates $J_{1}$ and $\sqrt{J_{2}}\left(J_{1}\right.$ : first invariant of stress tensor, $J_{2}$ : second invariant of stress deviator tensor) in the initial stress on the time axis, the analysis of load-carrying capacity is an instantaneous analysis under the $J_{1}$ and $\sqrt{J_{2}}$ at a certain point on the time axis. For example, even with the same concrete structure, the load-carrying capacity at the age of 30 days is different from the load-carrying capacity at the age of 1 year. In addition, even if the structure and age are the same, the load-carrying capacity differs depending on the method of concrete placement at the time of construction. If damage occurs due to, for example, an earthquake at a certain time, and then deteriorates due to environmental effects, the degree of deterioration is different from that without damage. In particular, in the instantaneous analysis of loadcarrying capacity, it is necessary to be uniformly reflected in the constitutive laws (stress-strain relationship) for concrete. Constituent laws for concrete have been studied for hardened concrete and concrete at an early age. Otherwise, it is impossible to carry out a coupled analysis. It is relatively simple to describe the time-dependent changes in the mechanical properties of concrete when the initial stress is zero. In case of an increase in strength in the presence of initial stress, no extensive studies have been carried out on the stress increment corresponding to the subsequent strain increment and its mathematical description. In the instantaneous analysis, it is necessary to consider the increase in strength according to the change in age and accurately establish the correspondence between the strain increment and stress increment from that point.

The present guidelines for controlling the cracking of mass concrete and standard specifications for concrete strictures do not even mention the coupled analysis of the initial stress and load-carrying capacity. Hence, it should be discussed in the revised committee to improve the guidelines.

\section{Conclusions}

This paper presents some of the control technologies of cracking for mass concrete in Japan, which have provided a remarkable progress. Many of the conventional control methods of cracking in Japan have been able to predict cracking relatively simply even at the site. Thus, civil engineers became more aware of the risk of cracking during construction and it was possible to better understand the measures to control cracking in advance. The remarkable progress in personal computers has made the large-capacity calculations relatively simple over the past 40 years. Furthermore, with the large improvements in the calculation speed and storage capacity, it is possible to analyse even 3D models with shapes close to an existing structure with tens of thousands of nodes. With this progress, it was possible to easily carry out a predictive analysis of cracking. In addition, Japan Concrete Institute provides software that incorporates the latest analysis methods for thermal stress analysis. Currently, only the Japanese version has been released [33]; the English version will be released soon.
This indicates that the actual stress behaviour with the volume change can be almost predicted if the used design values are appropriate. However, the design values used for the analysis vary depending on the used materials and mix proportions, curing conditions, environmental conditions, construction conditions, etc. In addition, an accurate quantification of the design values at an early age has not yet been established.

In the future, it will be necessary to obtain more precise and general-purpose mechanical and thermal properties. It will be also important to establish evaluation methods, particularly at an early age. Furthermore, it is necessary to study the various factors that affect the stress behaviours with volume changes. In the predictive analysis of stress behaviours and control of cracking with volume changes in concrete, as the development of analysis models and appropriate selection of design values for analysis conditions largely depend on the experience of the engineers carrying out the analysis, an easier analysis implies that it is necessary to provide more guidelines for engineers with a small analysis experience to not make major mistakes.

In RILEM, the thermal cracking issue has been actively discussed by the Technical Committee 254-CMS (Thermal Cracking of Massive Concrete Structures) to publish the stateof-the-art report [34]. The subsequent Technical Committee 287-CCS (Early age and Long-term Crack Width Analysis in RC Structures) have also discussed early-age cracking as well as long-term cracking. As the Japanese members, which would be involved in the revision of Guidelines for Control of Cracking of Mass Concrete, join the TC, collaborative work for cracking of mass concrete structures between RILEM and JCI is expected.

\section{Acknowledgements}

This work was financially supported by JSPS KAKENHI Grant Number $20 \mathrm{H} 02219$.

\section{Authorship statement (CRediT)}

Toshiaki Mizobuchi: Conceptualization, Resources, Writing original draft, Project administration.

Shingo Asamoto: Writing - review and editing, Funding acquisition.

\section{References}

[1] T. Tanabe, State of Research Activities on Thermal Stress Control of Mass Concrete. J Jpn Soc Civ Eng (1986) 372:1-16 (In Japanese). https://doi.org/10.2208/jscej.1986.372 1

[2] R. Sato, T. Kakazu, Outlines of "guidelines of practice for cracking control in mass concrete 2016". Concr J (2017) 55:1034-1041 (In Japanese). https://doi.org/10.3151/coj.55.12 1034

[3] S. Kajima, Pre-cooling system in mass concrete. Concr J (1988) 26:1320 (In Japanese). https://doi.org/10.3151/coj1975.26.5 13

[4] T. Sugi, Examples of measures of mass concrete. Concr J (1984) 22:1320 (In Japanese). https://doi.org/10.3151/coj1975.22.3 13

[5] R. Tsukayama, Thermal cracking in massive reinforced concrete structures. Cem Concr (1977) 370:11-17 (In Japanese).

[6] T. Mori, Thermal stress and degree of restraint of triangles and square plates with one side constrained. J Jpn Soc Civ Eng (1963) 89:45-56 (In Japanese). https://doi.org/10.2208/jscej1949.1963.45

[7] $\mathrm{ACl}$ Committee 207, Effect of Restraint, Volume Change, and Reinforcement on Cracking of Massive Concrete. ACl journal (1973) 70:445-470. https://doi.org/10.14359/11223 
[8] Japan Society of Civil Engineers, Japan Society of Civil Engineers Standard specifications for concrete structures - 1974 Fiscal year version, 1974 (In Japanese).

[9] Technical Committee on Thermal Stress of Mass Concrete, Proceedings of the $\mathrm{JCl}$ colloquium on the mechanism of thermal stress generation in massive concrete structures, Japan Concrete Institute, 1982 (In Japanese).

[10] Technical committee on Thermal Stress of Mass Concrete, Proceedings of the $\mathrm{JCl} 2$ nd colloquium on the mechanism of thermal stress generation in massive concrete structures, Japan Concrete Institute, 1984 (In Japanese).

[11] Technical Committee on JCl guidelines for control of cracking of mass concrete, guidelines for control of cracking of mass concrete, Japan Concrete Institute, 1986 (In Japanese).

[12] Japan Concrete Institute, Report of Technical Committee on Thermal Stress in Mass Concrete, 1985 (In Japanese).

[13] Japan Society of Civil Engineers, Japan Society of Civil Engineers Standard specifications for concrete structures - 1986 Fiscal year version, 1986 (In Japanese).

[14] P.B. Bamforth, Control of cracking caused by restrained deformation in concrete, CIRIA, C766. London, UK, 2018.

[15] ACl Committee 301, Specifications for Structural Concrete -An ACl standard, American Concrete Institute, 2016.

[16] Technical Committee on English version of JCl guidelines for control of cracking of mass concrete, guidelines for control of cracking of mass concrete 2008, Japan Concrete Institute, 2011.

[17] Autogenous shrinkage of Concrete Edited By Ei-ichi Tazawa. CRC Press, London, 1999.

[18] E. Tazawa, S. Miyazawa, T. Kasai, Chemical shrinkage and autogenous shrinkage of hydrating cement paste. Cem Concr Res (1995) 25:281287. https://doi.org/10.1016/0008-8846(95)00010-0

[19] Japan Society of Civil Engineers Standard specifications for concrete structures - 2012 Fiscal year version (Design), 2013.

[20] Technical Committee on English version of JCl guidelines for control of cracking of mass concrete, Guidelines for control of cracking of mass concrete 2016, Japan Concrete Institute, 2017.

[21] S.M.A. Nanayakkara, Cracking in concrete structures due to delayed Ettringite Formation. Transactions of the Society Structural Engineers, Sri Lanka, 2011, 17-22.

[22] L. Baingam, W. Saengsoy, P. Choktaweekarn, S. Tangtermsirikul, Diagnosis of a combined alkali silica reaction and delayed ettringite formation. ThammasatInt. J Sci Technol (2012) 17(4): 22-35.

[23] LCPC, Recommendations for preventing disorders due to delayed ettringite formation. Laboratoire Central des Ponts et Chaussées, Paris, France, 2009.

[24] M. Thomas, K. Folliard, T. Drimalas, T. Ramlochan, Diagnosing delayed ettringite formation in concrete structures. Cem Concr Res (2008) 38:841-847. https://doi.org/10.1016/j.cemconres.2008.01.003

[25] Japan Concrete Institute, Report of Technical committee on Thermal Stress of Mass Concrete, Identification of External Restraint Coefficient and Proposal of Calculation Method of Thermal Stress based on that Result, (1985):47-98 (In Japanese).

[26] Japan Society of Civil Engineers, Japan Society of Civil Engineers Standard specifications for concrete structures - 2007 Fiscal year version - design, 2007. Accessed 16 Mar. 2021. http://www.jsce.or.jp/committee/concrete/e/newsletter/std2007/t op.html

[27] Japan Concrete Institute, Report of Technical committee on Thermal Stress of Mass Concrete on Proposal for Calculation Method of Thermal Stress Crack Width, 1992 (In Japanese).

[28] T. Kamo, T. Tanabe, Study on method of calculating thermal crack width of mass concrete. Proc. of the Jpn Concr Inst (1989) 11: 457462 (In Japanese).

[29] Y. Tsuji, Fundamental study on the use of chemical pre-stress in concrete. J JCSE (1975) 235:111-124 (In Japanese). https://doi.org/10.2208/iscei1969.1975.235 111

[30] Y. Ishikawa, T. Tanabe, Unified calculation approach for varieties of chemical expansions in concrete based on the mechanical energy conservation hypothesis. J JSCE Div (2018) 74:119-138 (In Japanese). https://doi.org/10.2208/iscejmcs.74.119

[31] T. Tanabe, Y. Ishikawa, Chemical expansion effect in concrete and its numerical simulation based on the mechanical energy conservation hypothesis, CONCRACK5, 2017, 161-174.

[32] Y. Ishikawa, T. Tanabe, Theoretical development of CP method in predicting expansive cement concrete cracking, Fracture Mechanics of Concrete and Concrete Structures -Recent Advances in Fracture Mechanics of Concrete, 2010, 398-405.
[33] JCl Committee on Computer Code Development for Crack Control in Massive Concrete home page, http://www.jci-net.or.jp/ masscon/, (In Japanese) (accessed 16 Mar. 2021).

[34] E.M.R. Fairbairn, M. Azenha (Eds.), Thermal cracking of massive concrete structures: State of the art report of the RILEM Technical Committee 254-CMS. RILEM state-of-the-art reports 27, 2019, Springer. https://doi.org/10.1007/978-3-319-76617-1 